\title{
L'influence du « Capital maternel » sur la transmission intergénérationnelle de la langue oudmourte en république d'Oudmourtie
}

The influence of 'Maternal Capital' on Udmurt transgenerational language transmission in the Udmurt Republic

ВЛИЯНИЕ «МАТЕРИНСКОГО КАПИТАЛА» НА ПЕРЕДАЧУ УДМУРТСКОГО ЯЗЫКА

МЕЖДУ ПОКОЛЕНИЯМИ В УДМУРТСКОЙ РЕСПУБЛИКЕ

\section{Svetlana Russkikh}

\section{CpenEdition}

Journals

Édition électronique

URL : https://journals.openedition.org/efo/5682

DOI : $10.4000 /$ efo. 5682

ISSN : 2275-1947

\section{Éditeur}

INALCO

Édition imprimée

ISBN : 978-2-343-08571-5

ISSN : 0071-2051

\section{Référence électronique}

Svetlana Russkikh, «L'influence du « Capital maternel » sur la transmission intergénérationnelle de la langue oudmourte en république d'Oudmourtie », Études finno-ougriennes [En ligne], 47 | 2015, mis en ligne le 28 mars 2017, consulté le 20 septembre 2021. URL : http://journals.openedition.org/efo/5682 ; DOI : https://doi.org/10.4000/efo.5682

Ce document a été généré automatiquement le 20 septembre 2021.

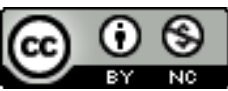

Études finno-ougriennes est mis à disposition selon les termes de la Licence Creative Commons Attribution - Pas d'Utilisation Commerciale 4.0 International. 


\section{L'influence du « Capital maternel » sur la transmission intergénérationnelle de la langue oudmourte en république d'Oudmourtie}

The influence of 'Maternal Capital' on Udmurt transgenerational language transmission in the Udmurt Republic

ВЛИЯНИЕ «МАТЕРИНСКОГО КАПИТАЛА» НА ПЕРЕДАЧУ УДМУРТСКОГО ЯЗЫКА

МЕЖДУ ПОКОЛЕНИЯМИ В УДМУРТСКОЙ РЕСПУБЛИКЕ

\section{Svetlana Russkikh}

Je remercie toutes les personnes qui, en Oudmourtie, ont accepté mes demandes d'entretiens. Je tiens aussi à remercier Cécile Lefêvre, Guillaume Enguehard et tout particulièrement Pierre-Yves Gaudard pour leurs relectures attentives et impliquées.

\section{Introduction}

1 Depuis l'année 2007, le gouvernement russe a mis en place une nouvelle politique démographique, dans le but d'encourager les familles à avoir plus d'enfants. Nous ne nous intéresserons pas ici de manière directe et générale à l'évaluation de cette politique, qui fait débat ${ }^{1}$. Néanmoins, il est possible de constater depuis ces dernières années une augmentation de la natalité en Russie, ainsi qu'en république d'Oudmourtie ${ }^{2}$ : entre 2006 et 2012, le nombre moyen d'enfants par femme (ISF) est passé en Russie de 1,4 à 1,7 et en république d'Oudmourtie de 1,3 à 1,94.

2 La visée de ce travail consiste plutôt à tenter d'évaluer les effets collatéraux de cette politique nataliste sur la transmission de la langue oudmourte. Il est en effet possible d'espérer un impact positif de cette augmentation des naissances sur le statut de la 
langue oudmourte. Pourtant, je montrerai qu'au contraire, cette politique nataliste, et particulièrement un de ces programmes («Capital maternel»), entraîne un changement de la structure familiale qui contribue à l'affaiblissement de la transmission de la langue menacée.

3 En effet, selon le dernier recensement réalisé en 2010, les Oudmourtes constituent une minorité dans leur propre république : au nombre de 410584 , ils ne représentent en effet que $28 \%$ de sa population ${ }^{5}$. En outre, il est établi depuis le recensement de 2002 que le nombre de locuteurs de l'oudmourte diminue. Alors qu'en 2002 leur nombre était estimé à 463837 personnes $^{6}$, ils ne sont plus que 324338 en $2010^{7}$. Cette diminution a même conduit l'Unesco à considérer la langue oudmourte comme menacée de disparition ${ }^{8}$.

4 Je fonderai mon analyse sur les données que j'ai recueillies au cours de deux expériences de terrain effectuées en république d'Oudmourtie ${ }^{9}$ en 2013 (2 mois) et en 2014 (1 mois). Dans une première partie, je pointerai le rôle de la grand-mère dans la transmission de la langue oudmourte. Dans la deuxième partie, je présenterai le programme "Capital maternel », mis en place en 2007. Je montrerai ensuite comment ce programme modifie la structure familiale, ce qui a pour conséquence d'affaiblir la transmission intergénérationnelle de la langue oudmourte.

\section{La transmission de la langue oudmourte et le rôle de la grand-mère}

5 Selon Galina Nikitina (2010), la famille joue un rôle central dans la transmission de la langue oudmourte. Pourtant, Natalija Il'ina et Natalija Kondrat'eva (2008) montrent que les parents oudmourtes utilisent principalement le russe pour communiquer avec leurs enfants. Cette étude sociologique, faite à Malaja Purga (village de 7734 habitants, où les Oudmourtes représentent $78,1 \%$ de la population) montre que seuls $15 \%$ des parents parlent oudmourtes avec leurs enfants (Il'ina, Kondrat'eva 2008, p. 338).

6 Par ailleurs, j'ai observé la même tendance pendant mon terrain auprès des familles oudmourtes. Le choix de la langue russe pour communiquer avec les enfants est motivé de diverses manières : (i). parce que les parents pensent aux difficultés qu'auront leurs enfants pour apprendre le russe, (ii). parce que l'un des conjoints ne parle pas l'oudmourte, ou (iii). parce que telle est leur habitude. Par exemple, Tatjana, une femme âgée de vingt-neuf ans, ne parle pas en oudmourte avec ses enfants car elle considère que cela peut les empêcher de bien parler le russe plus tard :

De toute façon cela ne sert à rien de parler oudmourte aujourd'hui. Il faut bien parler russe pour trouver un bon travail, réussir des études et réussir sa vie. C'est pour cette raison que je parle russe avec mes enfants. Si je parle avec eux en oudmourte, ils ne maîtriseront pas suffisamment bien le russe et ils ne vont pas réussir leurs études plus tard. Ils pourront toujours apprendre l'oudmourte par eux-mêmes, s'ils le souhaitent.

7 La transmission de la langue oudmourte ne passe donc pas automatiquement par les parents. En revanche, Marie Casen montre dans son travail de master que les étudiants oudmourtes d'Iževsk parlent oudmourte plus souvent avec leurs grands-mères qu'avec leurs parents (Casen 2010, p. 51-53). Dans les familles oudmourtes où la transmission ne passe pas par les parents, la langue est transmise principalement par la grand-mère, 
comme le montre cet extrait d'entretien avec Al'bina, une femme âgée de trente-et-un ans :

Dans notre famille, les enfants ne parlent en oudmourte qu'avec leur grand-mère (ma mère). On peut dire qu'ils ont grandi avec elle, elle a joué avec eux, elle a passé beaucoup de temps avec eux quand ils étaient tout petits. Moi et leur père, nous ne parlons avec eux qu'en russe. En fait, j'ai parlé toujours avec eux en russe et ma mère seulement en oudmourte. Je ne sais pas pourquoi, mais nous avons toujours fonctionné ainsi.

8 Les grands-mères assuraient traditionnellement la garde des enfants en bas âge et jouaient un rôle important dans leur éducation (Semenova 1996 [en ligne], Sorokin 2014, p. 78). Ce modèle de la grand-mère qui consacre sa vie à la famille et à ses petits-enfants est toujours répandu aujourd'hui. Selon Duprat-Kushtanina (2011), il existe surtout dans les milieux populaires, tandis que dans les milieux diplômés, la grand-mère tend à réduire son engagement au service de la famille. Cependant, la cohabitation intergénérationnelle peut valoriser la présence du modèle traditionnel pour les deux milieux sociaux. J'ai observé pendant mon terrain que dans certaines familles, les enfants jusqu'à l'âge de la scolarisation passent une grande partie de leur journée avec les grands-parents, notamment en raison de l'insuffisance des places disponibles dans les jardins d'enfants. En effet, la majorité des familles interrogées évoquent pendant les entretiens qu'obtenir une place au jardin d'enfant représente aujourd'hui un véritable problème. Cela les pousse donc à demander aux grands-parents de s'occuper de leurs enfants. Par exemple, c'est le cas de Nadja, une femme âgée de vingt-six ans :

Ma mère a gardé ma fille pendant quatre ans, le temps qu'on a attendu pour avoir une place au jardin d'enfant. Je ne pouvais pas la garder moi-même parce que je travaille. Ma mère travaille aussi mais ma grand-mère habite juste à côté, elle a donc beaucoup aidé.

9 Dans ces familles composées de trois générations, les enfants assimilent donc deux langues en même temps : ils apprennent le russe avec leurs parents et l'oudmourte avec leur grand-mère.

10 Toutefois, la mise en place de la politique nataliste et du programme «Capital maternel » conduit à la transformation de cette structure familiale.

\section{« Capital maternel » et décohabitation intergénérationnelle}

11 En 2006, dans son adresse annuelle à la nation ${ }^{10}$, Vladimir Poutine déclarait que l'immigration ne peut pas résoudre le problème démographique ${ }^{11} \mathrm{du}$ pays si le gouvernement ne crée pas les conditions favorables pour stimuler la natalité. Dans ce contexte, en 2007, un nouveau programme coûteux et original, nommé "Capital maternel », a été introduit ${ }^{12}$.

Ce « capital » est une allocation accordée au niveau fédéral pour les familles ayant un nouveau-né à condition qu'il ne s'agisse pas d'une primogéniture. Par ailleurs, ce capital ne peut être consacré qu'à l'amélioration des conditions de logement, à l'éducation des enfants ou à l'épargne-retraite de la mère. Aujourd'hui la somme allouée au titre du capital maternel représente 429408 roubles (soit environ 6 000 euros $)^{13}$. 
13 Selon les données du fonds des retraites, le Pensionnyj Fond $d^{14}$ (Godovoj otčet 2013, p. 45), la grande majorité des familles de la Fédération de Russie choisissent d'utiliser le capital maternel afin d'améliorer leurs conditions de logement. En république d'Oudmourtie aussi, cette tendance est dominante. En effet, de 2007 à 2012, 37003 familles ont utilisé le capital maternel pour le logement (30 531 pour rembourser un crédit immobilier et 6472 pour acquérir, construire ou reconstruire (agrandir) un logement), 586 pour l'éducation des enfants et seulement 10 familles pour l'alimentation d'un compte épargne-retraite pour la mère de famille ${ }^{15}$.

14 L'utilisation du capital maternel pour le logement entraîne parfois le déménagement des bénéficiaires et entraîne une décohabitation intergénérationnelle.

Parmi les personnes interrogées, la majorité a utilisé ou souhaite utiliser le capital maternel pour le logement afin de quitter le domicile de la belle-famille. C'est le cas de Rezeda, une femme âgée de vingt-deux ans, qui explique pendant l'entretien ce choix de déménager de chez sa belle-famille :

Tout de suite après le mariage on a commencé à vivre chez ma belle-famille. J'ai toujours voulu avoir mon propre logement, en fait. Comme on dit, il n'y a pas de place pour deux femmes dans la même cuisine. On a décidé d'utiliser le capital maternel pour acheter un appartement afin de ne plus vivre avec les parents de mon conjoint.

D'après les entretiens, les familles souhaitent déménager de chez leurs parents ou de chez leurs beaux-parents pour trois raisons principales: (i) conditions de logement insatisfaisantes ; (ii) conflits avec la belle-mère et (iii) problèmes avec l'alcool, ainsi que le montre le tableau (1).

Tableau 1

\begin{tabular}{|l|l|}
\hline $\begin{array}{l}\text { Conditions de } \\
\text { logement }\end{array}$ & $\begin{array}{l}\text { Je veux utiliser le capital maternel pour l'achat d'un logement parce que je suis } \\
\text { fatiguée de vivre chez les parents de mon mari... On n'a pas assez de place pour tout } \\
\text { le monde... On a } 30 \mathrm{~m}^{2} \text {, et on est six dans deux pièces, sachant que je suis enceinte et } \\
\text { qu'on va être encore plus nombreux (Nataša, vingt et un ans). }\end{array}$ \\
\hline $\begin{array}{l}\text { Conflits avec } \\
\text { la belle-mère }\end{array}$ & $\begin{array}{l}\text { J'ai toujours voulu avoir mon propre logement ; grâce au capital maternel, c'est le cas } \\
\text { aujourd'hui. Avant, on habitait chez la mère de mon mari et j'avais beaucoup de } \\
\text { conflits avec ma belle-mère (Rezeda, vingt-deux ans). }\end{array}$ \\
\hline $\begin{array}{l}\text { Problème } \\
\text { avec l'alcool }\end{array}$ & $\begin{array}{l}\text { On habite chez les parents de mon conjoint [...]. Au départ, la maison était plus petite, } \\
\text { on a donc construit cette pièce supplémentaire. Mais j'ai envie de vivre séparément à } \\
\text { cause du père de mon mari. Il boit souvent, c'est assez compliqué. Pour cette raison, } \\
\text { je pars souvent vivre chez mes parents avec mes enfants pour éviter les conflits et } \\
\text { tout ça... (Alena, vingt-six ans). }\end{array}$ \\
\hline
\end{tabular}

Pour ces familles, le capital maternel est donc le seul moyen de parvenir à échapper à la cohabitation intergénérationnelle, car sans cette aide proposée dans le cadre de la politique nataliste, elles n'ont pas la possibilité d'acquérir un logement, ou d'accéder à un crédit immobilier en raison du faible niveau de leurs revenus.

18 Ces familles achètent principalement un logement en milieu rural car le montant du capital maternel n'est pas suffisant pour acheter un logement en ville. Par exemple, Galina, une femme âgée de trente-neuf ans, a acheté avec le capital maternel une 
maison en bois à la campagne pour 300000 roubles, soit environ 4300 euros (voir photo) : «On a acheté une maison avec le capital maternel à la campagne [...], $69 \mathrm{~m}^{2}$, on a deux chambres, une cuisine. Avant on habitait chez ma belle-mère », explique Galina pendant l'entretien. La photo illustre le type de logement que les familles achètent à la campagne en ayant recours au capital maternel en république d'Oudmourtie.

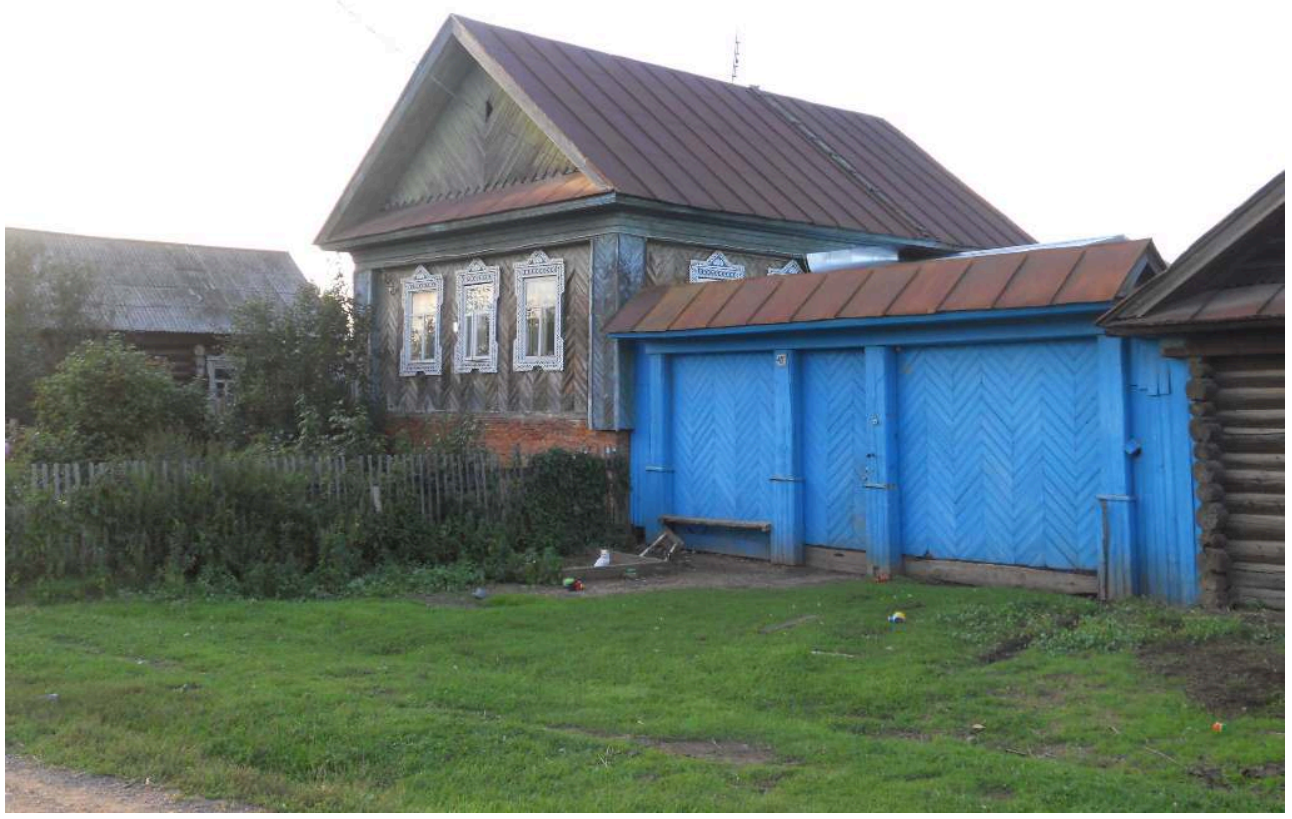

Photo prise pendant le terrain en république d'Oudmourtie (été 2013)

(c) Svetlana Russkikh

Il existe toutefois des exemples qui montrent que l'utilisation du capital maternel n'amène pas automatiquement à la décohabitation intergénérationnelle (Russkikh 2014 [en ligne]). Si les beaux-parents ou les parents ne peuvent plus vivre seuls, ils déménagent avec leurs enfants, comme nous pouvons le voir dans l'extrait d'entretien avec Polina, une femme âgée de trente ans :

On a construit une maison avec le capital maternel, on n'a pas encore complètement fini la construction mais on y vit déjà. Avant on habitait chez ma belle-mère, mais maintenant c'est elle qui vit chez nous, car elle ne peut plus vivre toute seule. Elle a besoin d'aide pour se laver, pour préparer à manger, pour prendre ses médicaments...

Cependant, contrairement à ce que semble indiquer ce dernier exemple, dans la majorité des cas, le recours au capital maternel s'oppose au maintien de la cohabitation intergénérationnelle : la famille élargie devient une famille nucléaire. Ce changement de la structure familiale bouleverse profondément les conditions de transmission de la langue oudmourte. 


\section{Affaiblissement de la transmission de la langue oudmourte comme conséquence de la décohabitation intergénérationnelle}

21 Après le déménagement, les enfants ne voient plus leurs grands-parents que pendant les grandes fêtes, les vacances scolaires ou au cours de visites occasionnelles. Diana, une femme de trente-et-un ans, raconte pendant notre entretien :

Après le mariage on a commencé à vivre chez les parents de mon mari. Grâce au capital maternel on a déménagé [...]. Les enfants vont chez leurs grands-parents l'été, pendant les vacances scolaires. Bien sûr, ils ne peuvent plus voir leurs grands-parents tous les jours, comme c'était avant. Oui, c'est triste, mais c'est comme ça.

Par ailleurs, les parents ne changent pas leurs habitudes linguistiques. Ils continuent à utiliser le russe comme langue principale de communication avec leurs enfants. Ainsi le fait de fréquenter les grands-parents plus rarement affaiblit la transmission de la langue oudmourte. Et si les grands-mères continuent à s'adresser à leurs petits-enfants en oudmourte, ceux-ci ont changé leur comportement linguistique. Après le déménagement, ils ne communiquent plus avec leurs grands-mères en oudmourte, mais en russe. Tamara, une femme de vingt-huit ans explique :

Ma mère continue à parler avec mes fils en oudmourte quand on vient chez elle. Mais les enfants commencent à oublier l'oudmourte, je crois. Bien sûr, ils comprennent leur grand-mère sans difficulté. Mais depuis qu'on a déménagé, ils répondent toujours en russe maintenant. En même temps, c'est normal, tout est en russe, l'école, les amis...

Il est intéressant de noter la même tendance au sein des familles tatares qui résident en république d'Oudmourtie, ainsi que le montre l'extrait d'entretien avec Gulnara, une femme âgée de vingt-sept ans :

Mon mari est russe, c'est peut-être pour ça que je n'ai jamais parlé tatar avec mes enfants. Quand on vivait chez ma mère, ils parlaient en tatar avec elle. Mais après le déménagement, j'ai l'impression qu'ils ont tout oublié. Je sais pas pourquoi, mais ils ont arrêté de parler en tatar aussi avec ma mère. Ça la rend triste.

Par ailleurs, les entretiens montrent que cet affaiblissement de la transmission concerne surtout des familles résidant chez les parents du mari, car les familles qui vivent chez les parents de la femme ont tendance à moins déménager. Dans ce dernier cas, les familles utilisent le capital maternel pour construire soit une pièce supplémentaire dans le but d'agrandir le logement initial, soit une nouvelle maison à côté de la maison familiale, comme le montre le cas de Roza, une femme âgée de trente ans :

Depuis plusieurs années, on vit chez ma mère. Au départ, on louait un petit appartement mais on avait des problèmes d'argent. Finalement, on a décidé de déménager chez ma mère. On a reçu 300000 roubles du capital maternel et on les utilise pour la construction d'une pièce supplémentaire. On va pratiquement doubler la superficie de la maison.

Cette tendance à utiliser le capital maternel afin d'améliorer les conditions de la cohabitation avec les parents de l'épouse s'explique en partie par le fait que la femme a moins de conflit avec sa mère qu'avec sa belle-mère.

L'éloignement et la recomposition de la famille élargie à la suite de l'utilisation du capital maternel pour le logement contribuent donc à remettre en cause l'une des 
possibilités de transmission de la langue oudmourte : la fréquentation assidue de la grand-mère par les petits-enfants.

\section{Conclusion}

L'utilisation de l'approche pluridisciplinaire permet de mieux comprendre les changements sociolinguistiques au sein de la société oudmourte. Cette étude montre de quelle manière la nouvelle politique démographique du gouvernement russe peut bouleverser la transmission de la langue.

La politique nataliste du programme «Capital maternel » exerce un effet négatif sur la transmission de la langue oudmourte, en milieu urbain aussi bien que rural. La décohabitation intergénérationnelle contribue au changement de la structure familiale, ce qui à son tour affaiblit la transmission de la langue oudmourte des grands-mères vers leurs petits-enfants. Autrement dit, la décohabitation intergénérationnelle à la suite de l'utilisation du capital maternel participe au processus de la russification des jeunes Oudmourtes, car leurs parents ne communiquent avec eux qu'en russe.

\section{BIBLIOGRAPHIE}

ARHANGELSKIJ 2014 = АРХАНГЕЛЬСКИЙ, В. Н., «ОЦЕНКА ДЕМОГРАФИЧЕСКОЙ РЕЗУЛЬТАТИВНОСТИ МЕР ПОМОЩИ СЕМЬЯМ С ДЕТЬМИ В РОССИИ», ДЕМОГРАФІЯ ТА СОЦІАЛЬНА ЕКОНОМІКА, n¹, С.108-120. CASEN Marie, 2010, « Les manifestations de l'identité oudmourte à Iževsk depuis 1985 », Mémoire de master, Inalco, Paris.

DUPRAT-KUSHTANINA Veronika, 2011, « Être grand-mère dans une ville russe. Une étape de parcours de vie de femmes », Recherches familiales, $n^{\circ} 8$, p. 81-90.

EŽOVA 2014 = ЕЖОВА Н. Н., «РОЖДАЕМОСТЬ В УДМУРТИИ И ЕЕ ЭТНИЧЕСКИЕ ОСОБЕННОСТИ», ЗДОРОВЬЕ, ДЕМОГРАФИЯ, ЭКОЛОГИЯ ФИННО-УГОРСКИХ НАРОДОВ, nº2, с.20-23.

Pensionnyj fond 2013 = ПЕНСИОННЫЙ ФОНД, ГОДОВОЙ ОТЧЕТ 2013, МОСКВА: ПЕНСИОННЫЙ ФОНД, $88 \mathrm{C}$.

IL'INA, KONDRAT’EVA 2008 = ИЛЬИНА Н. Б., КОНДРАТЬЕВА Н. В., «ФУНКЦИОНИРОВАНИЕ УДМУРТСКОГО ЯЗЫКА В СЕЛЕ МАЛАЯ ПУРГА УДМУРТСКОЙ РЕСПУБЛИКИ», Ünnepi írások Havas Ferenc tiszteletére, Budapest : A. Bereczki, M. Csepregi, L. Klima, 2008, с.336-344.

NIKITINA, HOR'KOVA 2011 = НИКИТИНА Г. А., ХОРЬКОВА Е. П., «УДМУРТСКАЯ РЕСПУБЛИКА - СУБЪЕКТ РОССИЙСКОЙ ФЕДЕРАЦИИ», БОТКИНА (éd.), ЭКОНОМИКА УДМУРТИИ: УЧЕБНОЕ ПОСОБИЕ, ИЖЕВСК: ИЗД-ВО «УДМУРТСКИЙ УНИВЕРСИТЕТ», С.7-37.

NIKITINA 2010 = НИКИТИНА Г. А, «ФИННО-УГОРСКИЕ НАРОДЫ РОССИИ В СОЦИАЛЬНЫХТРАНСФОРМАЦИЯХ ХХ ВЕКА: ОПЫТ И ПРОБЛЕМЫ АДАПТАЦИИ». ИНВОЖО=АЛЬМАНАХ УДМУРТСКОГО ПЕН-КЛУБА, n5-6, с.25-34. 
RUSSKIKH Svetlana, 2014, «L'impact de la nouvelle politique démographique russe sur la solidarité », Les Analyses du CERI [en ligne]. URL : http://www.sciencespo.fr/ceri/fr/content/ limpact-de-la-nouvelle-politique-demographique-russe-sur-la-solidarite

SEMENOVA 1996 = СЕМЕНОВА, В. В., «БАБУШКИ: СЕМЕЙНЫЕ И СОЦИАЛЬНЫЕ ФУНКЦИИ ПРАРОДИТЕЛЬСКОГО ПОКОЛЕНИЯ», СУДЬБЫ ЛЮДЕЙ: РОССИЯ ХХ ВЕК. БИОГРАФИИ СЕМЕЙ КАК ОБЪЕКТ СОЦИОЛОГИЧЕСКОГО ИССЛЕДОВАНИЯ, МОСКВА: ИНСТИТУТ СОЦИОЛОГИИ РАН, С.326-354.

SOROKIN 2014 = СОРОКИН Г. Г., «БАБУШКИ СОВРЕМЕННОЙ РОССИИ», ЖЕНЩИНА В РОССИЙСКОМ ОБЩЕСТВЕ, $\mathrm{n}^{\circ} 1, \mathrm{C.78-81.}$

ПОСЛАНИЕ ПРЕЗИДЕНТА ФЕДЕРАЛЬНОМУ СОБРАНИЮ РОССИЙСКОЙ ФЕДЕРАЦИИ 10 МАЯ 2006 годА.

ROSSTAT 2012 = РОССТАТ «СОЦИАЛЬНО-ДЕМОГРАФИЧЕСКИЙ ПОРТРЕТ РОССИИ: ПО ИТОГАМ ВСЕРОССИЙСКОЙ ПЕРЕПИСИ Н АСЕЛЕНИЯ 2010 ГОДА», ФЕДЕРАЛЬНАЯ СЛУЖБА ГОС. СТАТИСТИКИ, МОСКВА: ИИЦ «СТАТИСТИКА РОССИИ», 183 С.

VISHNEVSKIJ 2012 = ВИШНЕВСКИЙ, А. Г., «ДЕМОГРАФИЧЕСКИЙ ПРОРЫВ ИЛИ ДВИЖЕНИЕ ПО КРУГУ?», Demoscope, $\mathrm{n}^{\circ}$ 533-534 [en ligne]. URL : http://demoscope.ru/weekly/2012/0533/tema01.php

\section{NOTES}

1. Selon Anatolij Višnevskij (2012), cette politique démographique n'a pas eu d'impact sur la natalité, l'augmentation qu'on observe depuis ces dernières années résultant de la structure favorable des âges. En revanche, Vladimir Arhangel'skij (2014) considère que c'est bien grâce au programme «Capital maternel» que la natalité a augmenté. Selon Natalia Ežova (2014), le programme «Capital maternel » a eu un impact plus important, en matière de natalité, chez les Oudmourtes que chez les Russes.

2. La république d'Oudmourtie est un des sujets de la Fédération de Russie. Elle fait partie du district fédéral de la Volga et est située dans la partie occidentale du moyen Oural, entre les fleuves Kama et Vjatka. La république est composée de 25 régions, de 5 villes et de 3069 villages (Nikitina, Hor'kova 2011, p. 7).

3. Source : Rosstat de la Fédération de Russie, www.gks.ru

4. Source : Udmurtstat de la Fédération de Russie, http://udmstat.gks.ru/

5. En rusSe: ИНФОРМАЦИОННЫЕ МАТЕРИАЛЫ ОБ ОКОНЧАТЕЛЬНЫХ ИТОГАХ ВСЕРОССИЙСКОЙ ПЕРЕПИСИ НАСЕЛЕНИЯ 2010 ГОДА (Résultats finaux du recensement fait en 2010), http:// www.gks.ru/free_doc/new_site/perepis2010/perepis_itogi1612.htm, consulté le 14/10/2014.

6. Source: www.perepis2002.ru, http://www.perepis2002.ru/index.html?id=17, consulté le $30 / 01 / 2015$.

7. Rosstat, 2012, СОЦИАЛЬНО-ДЕМОГРАФИЧЕСКИЙ ПОРТРЕТ РОССИИ: ПО ИТОГАМ ВСЕРОССИЙСКОЙ ПЕРЕПИСИ НАСЕЛИЕНИЯ 2010 ГОДА, ФЕДЕРАЛЬНАЯ СЛУЖБА ГОСУДАРСТВЕННИЙ СТАТИСТИКИ, МОСКВА: «СТАТИСТИКА РОССИИ», 106.

8. Atlas of the World's Langages in Danger, www.unesco.org http://www.unesco.org/culture/ languages-atlas/index.php, consulté le 14/10/2014.

9. Pendant ces deux terrains, j'ai effectué 42 entretiens enregistrés et 12 non enregistrés. Ces entretiens ont été réalisés en république d'Oudmourtie, en milieu aussi bien urbain que rural : à Iževsk, capitale de la république, à Možga, ville moyenne et dans quatre villages des régions $\mathrm{dE}$ Možga et de Malaja Purga. Par souci d'anonymat, tous les prénoms cités dans cet article ont été changés. 
10. En russe : ПОСЛАНИЕ ПРЕЗИДЕНТА ФЕДЕРАЛЬНОМУ СОБРАНИЮ РОССИЙСКОЙ ФЕДЕРАЦИИ 10 МАЯ 2006 ГОДА (adresse annuelle du président de la Fédération de Russie à l'Assemblée fédérale, le 10 mai 2006), http://www.kremlin.ru/transcripts/9637, consulté le 14/10/2014.

11. La situation démographique en république d'Oudmourtie reflète la situation démographique générale de la Fédération de Russie et se caractérise par un faible niveau de fécondité (les générations suivantes sont moins nombreuses par rapport aux générations précédentes), un vieillissement de la population et une mortalité élevée, surtout chez les hommes d'âge actif.

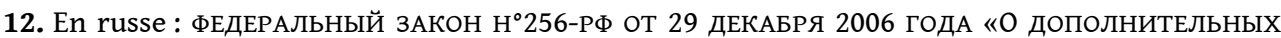
МЕРАХ ГОСУДАРСТВЕННОЙ ПОДДЕРЖКИ СЕМЕЙ, ИМЕЮЩИХ ДЕТЕЙ» (loi fédérale $n^{\circ} 256$, adaptée le 29 décembre 2006), http://www.consultant.ru/document/cons_doc_LAW_149064/, consulté le $14 / 10 / 2014$.

13. Cette somme n'est pas directement perçue par le bénéficiaire, elle est conservée par le Pensionnyj Fond (sauf dans le cas d'utilisation pour la construction ou pour la reconstruction du logement sans contrat avec une entreprise), et elle est reversée à la personne ou à l'organisme avec lesquels le bénéficiaire établit un contrat dans la limite des utilisations légales du capital maternel. Toute utilisation non prévue par le contrat ou encaissement direct du capital maternel sont interdits. Par ailleurs, les familles ne peuvent bénéficier du capital maternel qu'une seule fois.

14. En russe : ПЕНСИОНнЫЙ ФОНД.

15. Ces données statistiques ont été recueillies lors du terrain de 2013, auprès du Pensionnyj Fond de la république d'Oudmourtie qui se trouve à Iževsk.

\section{RÉSUMÉS}

La crise démographique, officialisée par le gouvernement russe en 2006, a contribué à la naissance d'une nouvelle étape pour la politique familiale en Russie. Le gouvernement instaure en 2007 une politique démographique essentiellement axée sur l'augmentation de la natalité.

Dans ce contexte, un programme coûteux et original, nommé «Capital maternel », a été mis en place. Cette étude portera sur le rôle central de la figure de la grand-mère dans la transmission de la langue oudmourte au sein des familles élargies. L'objectif de cet article est d'analyser de quelle manière l'introduction de la politique nataliste en 2007, à travers le programme «Capital maternel », entraîne un affaiblissement de cette transmission intergénérationnelle.

Demographic crisis, as it has been announced by Russian government in 2006 has induced an evolution in Russia's family policy. In 2007; a new family policy was launched, aimed at increasing birth rate. Its main tool was a new, costly and original programme called "Maternal Capital".

This study focuses on the central role of the grandmother in Udmurt language transmission within extended families. Its aim is to analyse how the implementation of the new Russian demographic policy of 2007, through the programme called "Maternal Capital", induces a weakening of this transgenerational transmission.

ДЕМОГРАФИЧЕСКИЙ КРИЗИС, ОБЪЯВЛЕННЫЙ РОССИЙСКИМ ПРАВИТЕЛЬСТВОМ В 2006 ГОДУ, СПОСОБСТВОВАЛ ПОЯВЛЕНИЮ НОВОГО ЭТАПА В СЕМЕЙНОЙ ПОЛИТИКЕ РОССИИ. В РЕЗУЛЬТАТЕ, В 2007 ГОДУ ГОСУДАРСТВО УТВЕРДИЛО НОВУЮ ДЕМОГРАФИЧЕСКУЮ ПОЛИТИКУ, ГЛАВНЫМ 
ОБРАЗОМ НАПРАВЛЕННУЮ НА ПОВЫШЕНИЕ РОЖДАЕМОСТИ. В РАМКАХ ЭТОЙ ПОЛИТИКИ, БЫЛА СОЗДАНА ПРИНЦИПИАЛЬНО НОВАЯ ПРОГРАММА, НАЗВАННАЯ «МАТИРИНСКИМ КАПИТАЛОМ». ДАННОЕ ИССЛЕДОВАНИЕ ЗАТРАГИВАЕТ ЗНАЧИМОСТЬ РОЛИ, КОТОРУЮ ИГРАЕТ БАБУШКА, КАК ЧЛЕН СЕМЬИ, В ПЕРЕДАЧЕ УДМУРТСКОГО ЯЗЫКА В РАСШИРЕННЫХ СЕМЬЯХ. ТАКИМ ОБРАЗОМ, ЦЕЛЬЮ ЭТОЙ СТАТЬИ ЯВЛЯЕТСЯ АНАЛИЗ ТОГО, КАК ПРОНАТАЛИСТСКАЯ ПОЛИТИКА, ВВЕДЕННАЯ В 2007 ГОДУ, СПОСОБСТВУЕТ - ПОСРЕДСТВОМ ПРОГРАММЫ МАТЕРИНСКОГО КАПИТАЛА ОСЛАБЛЕНИЮ ПЕРЕДАЧИ УДМУРТСКОГО ЯЗЫКА МЕЖДУ ПОКОЛЕНИЯМИ.

\section{INDEX}

Index géographique : Fédération de Russie, Iževsk, Kama, Malaja Purga, Možga, Oudmourtie, Oural, Vjatka

Index chronologique : XXIe siècle

Mots-clés : démographie, cohabitation intergénérationnelle, natalité, politique démographique, transmission linguistique

motscleset demograafia, keele edasiandmine, mitmepõlvkonna kooselu, rahvastiku poliitika, sündimus

nomsmotscles Oudmourtes, Russes

motsclesru ДЕМОГРАФИЯ, ДЕМОГРАФИЧЕСКАЯ ПОЛИТИКА, РОЖДАЕМОСТЬ, ПЕРЕДАЧА ЯЗЫКА, СОЖИТЕЛЬСВО НЕСКОЛЬКИХ ПОКОЛЕНИЙ

Thèmes : sociologie

Keywords : demography, intergenerational coexistence, birth rate, demographic policy, language transmission 